\title{
ANÁLISE CONCEITUAL DE LINHAS DE TRANSMISSÃO OPERANDO EM REGIME PERMANENTE SENOIDAL ${ }^{1}$
}

\author{
Cíntia Arantes Silva ${ }^{2}$ \\ Gustavo Lobato Campos ${ }^{3}$ \\ Mariana Guimarães dos Santos ${ }^{4}$ \\ Mário Luiz Rodrigues Oliveira ${ }^{5}$ \\ Rafael Vinicius Tayette da Nobrega ${ }^{6}$
}

\begin{abstract}
RESUMO
Este artigo apresenta a operação de linhas de transmissão operando em regime permanente senoidal, a relação dos acoplamentos elétricos (condutivo e capacitivo) e magnéticos (indutivo). A avaliação de tais acoplamentos é de fundamental importância, uma vez que estão diretamente associados, por exemplo, com as perdas nas linhas, a segurança e a confiabilidade do sistema elétrico interligado e a segurança de terceiros quando expostos aos campos eletromagnéticos ao longo da faixa de servidão.
\end{abstract}

Palavras-chave: Interferência eletromagnética. Linha de transmissão. Acoplamentos elétricos. Acoplamentos magnéticos.

\section{INTRODUÇÃO}

A demanda por energia elétrica vem crescendo constantemente desde o começo do século XX, devido tanto ao aumento da população quanto ao seu crescente acesso a equipamentos eletroeletrônicos. Atualmente, a maneira mais eficiente de levar energia do ponto de geração aos consumidores finais é por meio de linhas de transmissão (LT’s), que são componentes fundamentais do Sistema Elétrico de Potência. No entanto, devido a problemas

\footnotetext{
${ }^{1}$ Como citar este artigo: ARANTES, Cíntia et al. Análise conceitual de linhas de transmissão operando em regime permanente senoidal. ForScience: revista científica do IFMG, Formiga, v. 4, n. 2, e00206, jul/dez 2016.

${ }^{2}$ Graduanda em Engenharia Elétrica pelo Instituto Federal de Educação, Ciência e Tecnologia de Minas Gerais (IFMG), Campus Formiga. (http://lattes.cnpq.br/7351351413637001). E-mail: cintiaarantess@ hotmail.com.

${ }^{3}$ Doutorando em Engenharia Nuclear pela Universidade Federal de Minas Gerais. Professor do IFMG - Campus Formiga. (http://lattes.cnpq.br/9613750934178733). E-mail: gustavo.lobato@ifmg.edu.br.

${ }^{4}$ Mestre em Engenharia Elétrica - Universidade Federal de São João Del Rei (UFSJ/CEFET). Professora efetiva do IFMG - Campus Formiga. (http://lattes.cnpq.br/4661598896242893). E-mail: mariana.santos@ifmg.edu.br.

${ }^{5}$ Especialista em Design Instrucional para EaD Virtual pela Universidade Federal de Itajubá (2013). Professor do IFMG - Campus Formiga. (http://lattes.cnpq.br/8279467349228894). E-mail: mario.luiz@ifmg.edu.br.

${ }^{6}$ Doutorando em Engenharia Elétrica - Universidade de São Paulo (USP). Professor do IFMG - Campus Formiga. (http://lattes.cnpq.br/7492232498866714). E-mail: rafael.nobrega@ifmg.edu.br.
} 
geográficos, econômicos ou sociais, algumas linhas de transmissão passam próximas a áreas residenciais, causando preocupação na comunidade local quanto aos danos à saúde que os elevados campos elétricos e magnéticos presentes nessas LT's podem causar (COSTA; MONTEIRO, 2013).

Níveis elevados de campos eletromagnéticos podem provocar danos a seres humanos, animais ou objetos localizados próximos às linhas de transmissão, sendo assim necessária a regulamentação de faixas de servidão ao longo das trajetórias das linhas, para a inibição máxima do recebimento dos campos eletromagnéticos. Este artigo tem como finalidade conceituar o funcionamento de uma linha de transmissão aérea e analisar a interferência eletromagnética de sua operação em regime permanente, na frequência industrial de $60 \mathrm{~Hz}$, devido aos campos elétricos e magnéticos.

\section{GERAÇÃO, TRANSMISSÃO E DISTRIBUIÇÃO DE ENERGIA ELÉTRICA}

O setor elétrico é basicamente divido em geração, transmissão e distribuição. Em uma visão simples, podem-se definir esses setores da seguinte forma:

- Geração: setor responsável pela transformação de qualquer tipo de energia em energia elétrica - na maioria das vezes, essa energia é produzida nas Centrais de usinas hidrelétricas, devido ao nosso principal recurso ser hídrico.

- Transmissão: setor que faz com que a energia produzida chegue aos centros de distribuição.

- Distribuição: setor que faz a entrega da energia elétrica gerada ao consumidor final, isto é, para as áreas residenciais, comerciais, industriais e rurais.

A energia elétrica, na maioria das vezes, é produzida distante dos grandes centros de consumo; com isso, é preciso levá-la até eles. Por uma questão econômica, são necessários transformadores para abaixar e elevar a tensão, quando necessário. Por exemplo, na saída da hidrelétrica, um transformador eleva a tensão, de modo a evitar maiores perdas durante o processo de transmissão. Nas intermediações dos centros urbanos existem as subestações, que irão abaixar essa tensão e, então, fazer a distribuição aos consumidores residenciais e industriais em níveis adequados. A transmissão da energia aos centros urbanos e industriais é realizada mediante as linhas de transmissão, foco deste trabalho (PEREIRA JUNIOR, 2013). 


\section{LINHAS DE TRANSMISSÃO}

Uma linha de transmissão é o meio mais importante para entregar a energia produzida aos consumidores finais. Definem-se linhas de transmissão como sistemas físicos que transportam um sinal elétrico entre um gerador e uma carga através de um campo eletromagnético (HAYT, 1983). Existem alguns modelos de linhas de transmissão, como linhas subterrâneas, linhas áreas e linhas mistas. Este artigo estuda somente as linhas de transmissão aéreas em corrente alternada, ou linhas de transmissão CA.

Para evitar principalmente as perdas elétricas na transmissão de energia ao longo do trajeto até os centros distribuidores, a transmissão de energia elétrica utiliza níveis de tensões elevadas, e é justamente de acordo com o nível de tensão a que estão submetidas que as linhas são classificadas.

Há determinadas grandezas que influenciam o nível de tensão aplicada a uma linha de transmissão, como, por exemplo: a potência transportada, o comprimento da linha e o custo de instalação. Destaca-se, ainda, que existem regulamentações dos órgãos competentes que estabelecem os níveis de tensão a serem aplicados. Atualmente, no Brasil, os níveis para transmissão em corrente alternada são: $750 \mathrm{kV}, 500 \mathrm{kV}, 440 \mathrm{kV}, 345 \mathrm{kV}$ e $230 \mathrm{kV}$, e para subtransmissão, tem-se $138 \mathrm{kV}$ e $69 \mathrm{kV}$ Operador Nacional do Sistema (ONS) (OLIVEIRA, 2009).

\section{COMPONENTES DAS LINHAS DE TRANSMISSÃO}

O desempenho elétrico das linhas de transmissão depende quase exclusivamente de suas características físicas e da topologia do sistema interligado. Elas não só ditam o seu comportamento em regime normal de operação, definindo seus parâmetros elétricos, como também, quando em condições adversas, como limites no fluxo de energia ao longo das linhas.

O transporte de energia elétrica pelas linhas de transmissão tem a função, perante o setor elétrico, de ser eficiente, confiável e econômico. Para se transportar uma determinada quantidade de energia elétrica a uma distância preestabelecida, há um número grande de variáveis associadas à linha que são relevantes (LABEGALINI et al., 1992):

- Valor da tensão de transmissão;

- Número, tipo, bitolas e disposição dos cabos condutores; 
- Número e tipo dos isoladores e distâncias de segurança;

- Número de circuitos trifásicos;

- Materiais estruturais e a forma dos suportes resistirem aos esforços, etc.

Todos os fatores intervenientes estão relacionados com os componentes físicos das linhas. Uma linha de transmissão compõe-se das seguintes partes principais:

- Cabos condutores de energia e acessórios: são os elementos ativos das linhas de transmissão. Atuam com a função de "guias" da energia elétrica, já que, como explica a teoria da transmissão, os reais agentes do transporte da energia elétrica são os campos elétricos e os campos magnéticos. A escolha adequada e o dimensionamento correto dos cabos condutores são de extrema importância para que as perdas de energia elétrica possam ser mantidas, economicamente, dentro dos limites toleráveis (por efeito Joule ou por Corona) e para se controlar os níveis de rádio interferência e ruídos acústicos. Os condutores ideais para linhas aéreas de transmissão são aqueles que puderem apresentar as seguintes características:

1. Alta condutibilidade elétrica;

2. Baixo custo de confecção, instalação e manutenção (mais importante);

3. Boa resistência mecânica;

4. Baixo peso específico;

- Alta resistência à oxidação e à corrosão por agentes químicos poluentes.

Porém, as condições acima mencionadas infelizmente são de "condutores ideais", pois não são atendidas simultaneamente por nenhum material em particular. Mas os que atendem ao maior número dessas propriedades são os condutores de cobre e de alumínio (atualmente predominante no Brasil) (LABEGALINI et al., 1992).

- Estruturas isolantes: os cabos são suportados pelas estruturas através dos isoladores, que, como o próprio nome diz, os mantêm isolados eletricamente do seu suporte e do solo. Devem resistir tanto às solicitações mecânicas quanto às elétricas. As solicitações mecânicas a que os isoladores são submetidos pelos cabos condutores são:

1. Forças verticais - devido ao próprio peso; 
2. Forças horizontais axiais;

3. Forças horizontais transversais.

As solicitações de natureza elétrica a que um isolador deve resistir são as tensões mais elevadas que podem ocorrer em algum momento nas linhas, sendo:

4. Sobretensões de origem atmosférica, cujas intensidades podem ser muito elevadas e variadas;

5. Surtos de sobretensão de manobra ou chaveamento, que são de curta duração, podendo atingir de três a cinco vezes a tensão normal de operação;

6. Tensão normal ou sobretensões senoidais.

Suas superfícies devem ter acabamento capaz de resistir bem às exposições ao tempo. Assim, em sua fabricação empregam-se os seguintes materiais: porcelana vitrificada, vidro temperado ou material sintético composto. Em linhas de transmissão, são utilizados basicamente três tipos de isoladores:

$\checkmark$ Isoladores de pino;

$\checkmark$ Isoladores tipo pilar;

$\checkmark$ Isoladores de suspensão.

- Estruturas de suporte: também chamadas de elementos de sustentação dos cabos das linhas de transmissão. A quantidade de pontos de suspensão na estrutura depende da quantidade de cabos condutores e cabos para-raios a serem suportados. Há uma grande variedade de estruturas em uso, e suas dimensões e formas dependem dos seguintes fatores (LABEGALINI et al., 1992):

1. Disposição e distância dos condutores - disposição triangular, horizontal ou vertical;

2. Dimensões e formas de isolamento - são determinadas pela tensão nominal e pelas sobretensões previstas;

3. Flechas dos condutores;

4. Altura de segurança;

5. Função mecânica - determinada pelos tipos de carga que devem suportar;

6. Formas de resistência das estruturas - estruturas autoportantes (rígidas, flexíveis, mistas ou semirrígidas) ou estruturas estaiadas; 
7. Materiais estruturais - estruturas metálicas, madeiras ou estruturas de concreto;

8. Número de circuitos etc.

A forma final dos suportes decorre da "arquitetura" mais adequada, com base em todos os requisitos citados acima, buscando sempre o melhor custo/benefício. A FIG. 1 mostra alguns exemplos de estruturas.
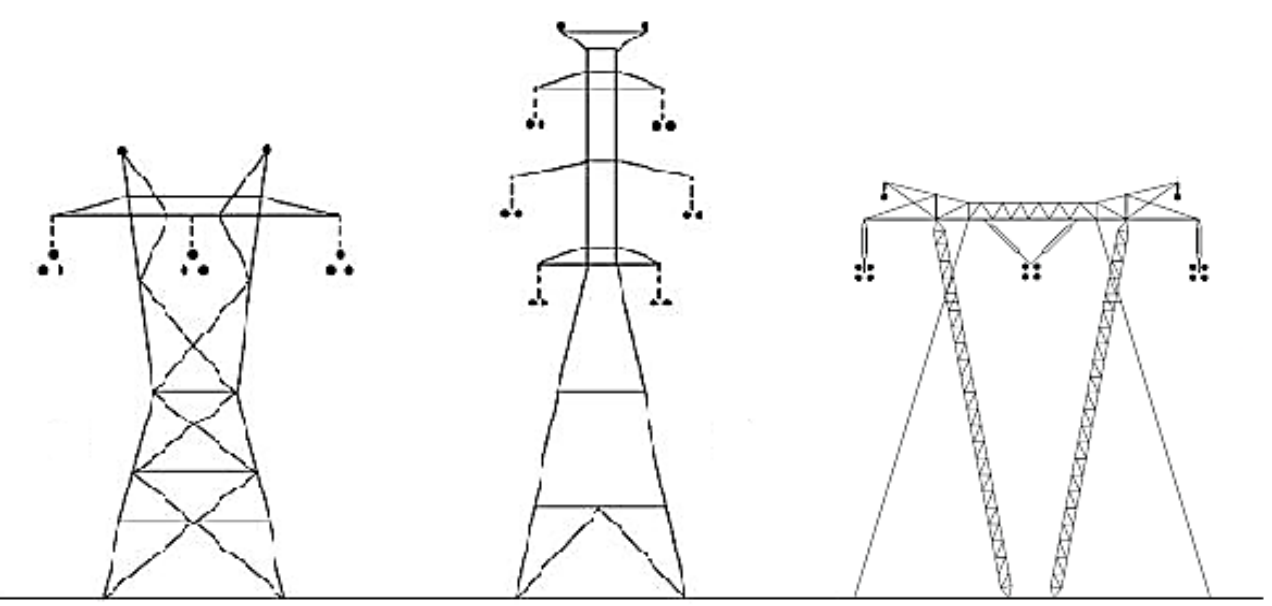

Figura 1- Estruturas de torres de LTs do tipo delta, pirâmide e estaiada em V, respectivamente Fonte: Lima (2013).

- Cabos de guarda ou para-raios: ocupam a parte superior das estruturas e têm a função de interceptar as descargas atmosféricas e evitar que atinjam os condutores, reduzindo assim a possibilidade de ocorrência de interrupções no sistema (LABEGALINI et al., 1992).

Os principais elementos de uma linha de transmissão podem ser vistos na FIG. 2.

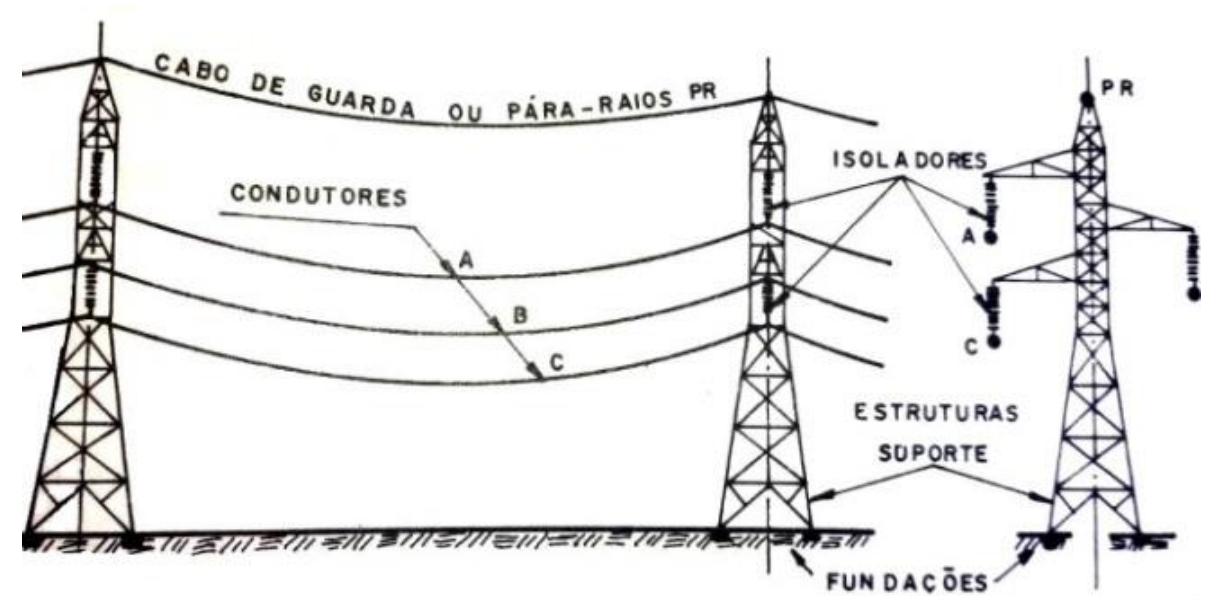

Figura 2 - Tipos de suportes aplicados nas Linhas de Transmissão Fonte: Labegalini et al. (1992).

ForSci.: r. cient. IFMG, Formiga, v. 4, n. 2, e00206, ago./dez. 2016. 


\section{OPERAÇÃO DE UMA LINHA DE TRANSMISSÃO}

As linhas de transmissão têm sua operação modelada pelas grandezas elétricas resistência, indutância e capacitância, consideradas uniformemente distribuídas ao longo da linha. A resistência relaciona-se com a perda de energia, uma vez que a potência dissipada é dada pela Equação 1:

$$
P=R \times I^{2}
$$

Onde:

- $\mathrm{P}=$ Potência dissipada, dada em W.

- $\mathrm{R}=$ Resistência, dada em $\Omega$.

- $\mathrm{I}=$ Corrente, dada em A.

A indutância armazena energia magnética por causa da variação da corrente (CA). A capacitância armazena energia elétrica devido à diferença de potencial entre linha e solo (FUCHS, 1977).

O comportamento de uma linha de transmissão pode ser divido em dois regimes de análise:

- Regime permanente: operação na frequência de $60 \mathrm{~Hz}$.

- Regime transitório: o espectro de frequência pode chegar até $10 \mathrm{MHz}$ devido às manobras na linha de transmissão (operações de chaveamento), curtoscircuitos nas linhas ou elementos que compõem o sistema, ou, ainda, em decorrência das descargas atmosféricas.

\section{INTERFERÊNCIA ELETROMAGNÉTICA DEVIDO À OPERAÇÃO DE UMA LINHA DE TRANSMISSÃO}

De modo geral, a avaliação da interferência eletromagnética das linhas de transmissão se dá em função do regime de operação da linha (permanente ou transitório) e de qual dispositivo é afetado. Normalmente, pode-se abordar este complexo problema em duas partes:

Linha de transmissão em regime permanente: a interferência é decomposta nos acoplamentos elétricos (condutivo e capacitivo) e magnéticos (indutivo).

- Acoplamento condutivo: modelagem longitudinal - projeta-se ao longo da linha (ligado ao efeito resistivo). 
- Acoplamento capacitivo: modelagem divergente - projeta-se ao longo do solo, como um efeito transversal à linha.

- Acoplamento indutivo: modelagem rotacional - projeta-se ao longo da linha.

Linha de transmissão em regime transitório: neste caso, a interferência eletromagnética deve ser avaliada em função do acoplamento eletromagnético (irradiado), principalmente na faixa superior do espectro. Assim, os efeitos dos campos elétrico e magnético não podem ser analisados de forma independente, tornando o problema extremamente complexo (SANTOS, 2011).

\section{CAMPOS ELÉTRICOS E MAGNÉTICOS PRODUZIDOS POR LINHAS DE TRANSMISSÃO}

O caráter vetorial dos campos elétricos e magnéticos tem de ser levado em consideração na realização de medições e cálculos realizados. Estes campos são representados por vetores que possuem magnitude, direção e sentido. Os princípios fundamentais do eletromagnetismo afirmam que cada carga individual cria um campo elétrico, de forma tal que as linhas de campo saem das cargas positivas e chegam às negativas, sendo este campo capaz de gerar forças sobre as cargas elétricas sob sua influência. Pode-se ainda afirmar que o campo elétrico local (em um determinado ponto) é dado pela soma vetorial dos campos elétricos gerados por todas as cargas existentes, e a intensidade do campo é inversamente proporcional ao quadrado da distância à fonte.

Por sua vez, o campo magnético é gerado por cargas elétricas em movimento, e sua direção é perpendicular à direção do movimento das cargas. O campo magnético local é a soma vetorial de seus diversos componentes, e sua magnitude também é inversamente proporcional à distância da fonte (GUEDES, 2011).

A FIG. 3 ilustra as formas típicas dos perfis de campo elétrico vertical e magnético resultante, gerados por uma linha de transmissão. Como pode ser observado, o campo magnético e o campo elétrico possuem valor máximo diretamente abaixo da linha, decaindo rapidamente com a distância em relação aos condutores (KUSTER, 2011).

Sabe-se que o nível de campo magnético gerado por uma linha de transmissão depende dos seguintes fatores:

- Corrente na linha: quanto maior a corrente, maior o campo magnético. 
- Altura dos condutores: condutores mais baixos resultam em um nível maior de campo magnético.

- Configuração das fases: o decaimento do campo magnético resultante é função do arranjo dos condutores - para linhas com fases transpostas ao longo do trajeto, o campo magnético decresce com o inverso do quadrado da distância; para linhas com fases não transpostas, o campo magnético diminui com o inverso do cubo da distância.

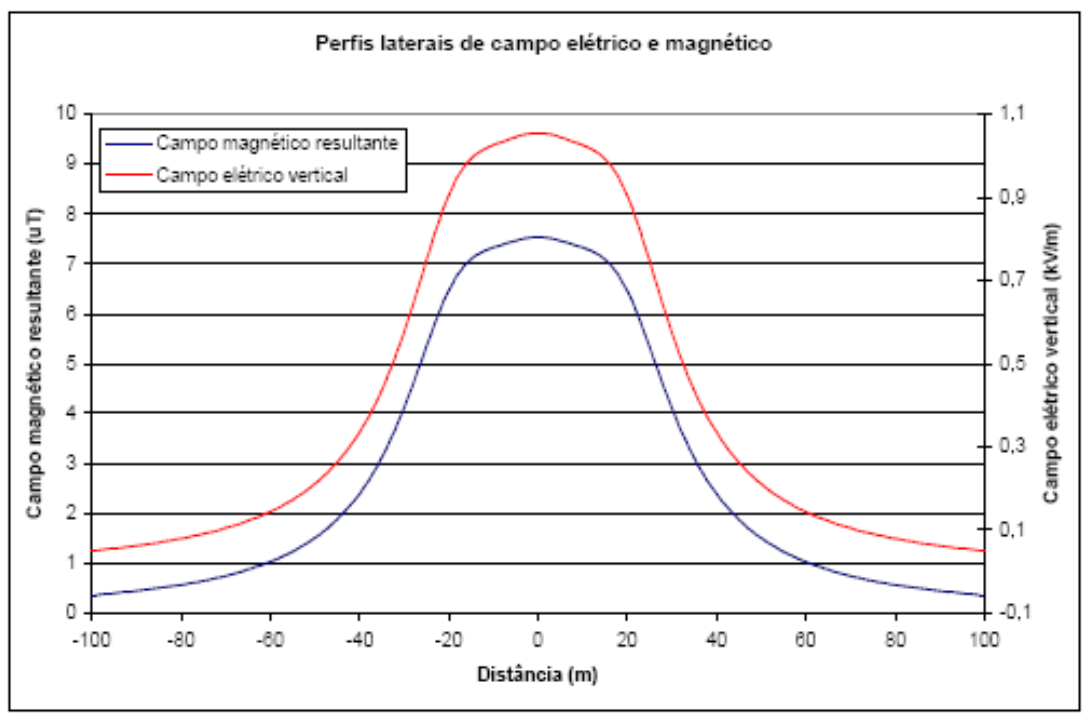

Figura 3 - Tipos de suportes aplicados nas Linhas de Transmissão Fonte: Kuster (2011).

De forma similar, o campo elétrico produzido por uma linha de transmissão é função dos seguintes parâmetros:

- Tensão da linha: quanto maior a tensão, maior o campo elétrico.

- Altura dos condutores: condutores mais baixos resultam em um nível maior de campo elétrico.

- Configuração e número de condutores: influenciam a taxa de decaimento do campo elétrico em função da distância em relação à linha.

- Presença de objetos próximos: objetos próximos ao ponto de medição, como árvores e estruturas metálicas, interferem na medição do campo elétrico.

É importante observar que o campo magnético gerado por uma linha de transmissão não é constante ao longo do tempo, uma vez que a corrente na linha muda em função das 
variações de carga. O campo elétrico, por outro lado, é constante ao longo do tempo, já que é função da tensão da linha, a qual varia muito pouco.

Os campos eletromagnéticos podem ser calculados pelas equações de Maxwell e/ou aplicações das Leis de Gauss, Faraday-Lenz e de Ampére, ou, ainda, através de medições. Os campos elétricos e magnéticos são acoplados, isto é, se um é conhecido, o outro pode ser calculado a partir deste, sendo uma difícil tarefa em alguns casos. Mas, como já citado, a proposta deste artigo é o estudo da linha de transmissão em regime permanente, no qual os campos elétricos e magnéticos podem ser considerados independes e desacoplados. Os campos próximos de linhas de transmissão (a variação é lenta com o tempo) permitem simplificações que desacoplam as equações de Maxwell, possibilitando o cálculo a partir das equações da quase estática (GUIMARÃES, 2005). Ou seja, considerando-se todos os termos que possuem $d / d t=0$, as Equações de Maxwell assumem as seguintes formulações, mostradas nas Equações 2, 3, 4 e 5.

$$
\begin{aligned}
& \vec{\nabla} \cdot \vec{E}=\rho \\
& \vec{\nabla} \times \vec{E}=0 \\
& \vec{\nabla} \cdot \vec{B}=0 \\
& \vec{\nabla} \times \vec{B}=0+\vec{J}
\end{aligned}
$$

\section{FAIXA DE SERVIDÃO}

A norma brasileira NBR 5422 - "Projeto de linhas aéreas de transmissão de energia elétrica" - especifica as condições básicas de um projeto de linhas aéreas de transmissão, de modo a garantir níveis mínimos de segurança e limitar perturbações em instalações próximas (ABNT, 1985). O estabelecimento destes limites de segurança para exposição de campos eletromagnéticos sobre ambos os lados das linhas ao longo de sua trajetória é denominado: "faixa de servidão" ou "faixa de passagem". Dentre outros parâmetros, a norma estabelece uma metodologia para o cálculo da largura da faixa de servidão de uma linha aérea de transmissão. No caso de um único circuito, a largura mínima L da faixa é dada pela Equação 6 abaixo. 


$$
L=2 \times(b+d+D)
$$

Onde:

- b - Distância horizontal do eixo do suporte ao ponto de fixação do condutor mais afastado deste eixo.

- d - Soma das projeções horizontais da flecha do condutor e do comprimento da cadeia de isoladores após seu deslocamento angular devido à ação do vento.

- D - Dado por DU /150, valor mínimo de 0,5 m.

- $\mathrm{D}_{\mathrm{U}}$ - Tensão máxima de operação da linha, em kV.

A FIG. 4 mostra uma linha de transmissão com os parâmetros L, b, d e D.

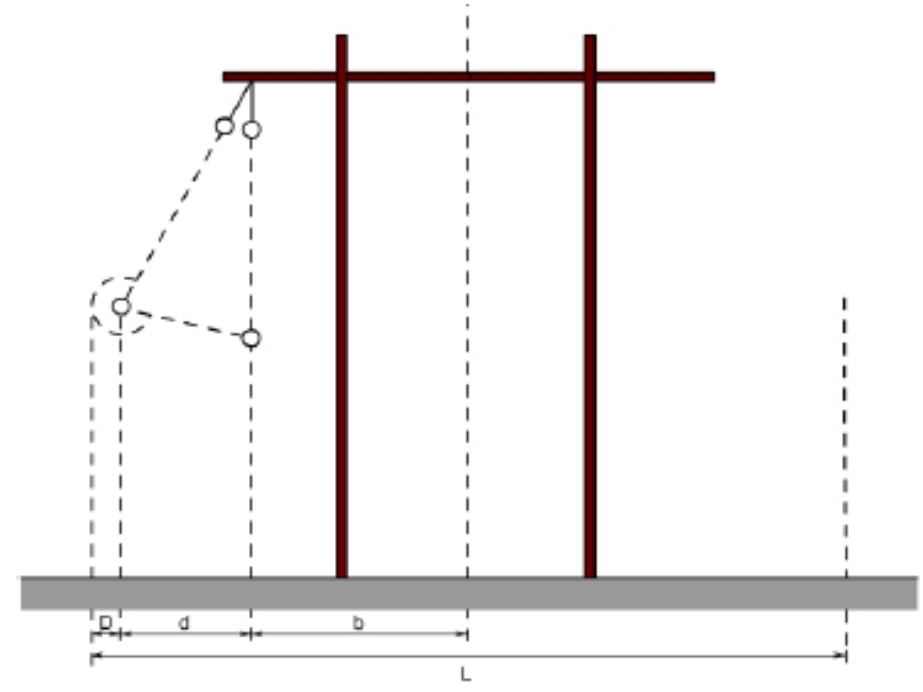

Figura 4 - Cálculo da largura da faixa de servidão de uma linha de transmissão Fonte: Kuster (2011).

\section{AVALIAÇÃO DOS LIMITES E EFEITOS DA EXPOSIÇÃO HUMANA A CAMPOS ELETROMAGNÉTICOS}

A associação entre a exposição a campos eletromagnéticos gerados pelas linhas de transmissão e seus possíveis riscos à saúde humana é um assunto que tem recebido grande atenção dos pesquisadores nas últimas décadas (HAVAS, 2004), (MORENO, 2005), (PERRO, 2007), (POLK, 1991) e tem se tornado fator relevante em projetos de construção ou ampliação de sistemas de transmissão. Trata-se de um tema polêmico, pois tem gerado controvérsia na comunidade científica, assim como preocupação junto ao público em geral. 
Um dos primeiros trabalhos nesta área foi o estudo epidemiológico conduzido em Denver - EUA (WERTHEIMER; LEEPER, 1979), que indicou uma relação entre a incidência de câncer em crianças (leucemia) e a proximidade de suas casas a linhas de transmissão de energia elétrica (LT’s) (RAMÍREZ, 2001). Entretanto, depois deste estudo inicial, vários outros estudos epidemiológicos e experimentais têm avaliado as possíveis relações entre câncer (gerados por campos eletromagnéticos) em humanos, animais de laboratório e em cultura de células (SANTOS, 2011). Abaixo, algumas conclusões desses trabalhos (MORENO, 2001):

- A exposição de seres humanos e animais a campos elétricos e magnéticos em $60 \mathrm{~Hz}$ induzem correntes elétricas internas. A densidade da corrente induzida é não uniforme em todo o corpo;

- Os campos elétricos de frequência 50 ou $60 \mathrm{~Hz}$ possuem baixa capacidade de penetração; a grande maioria dos efeitos biológicos está associada principalmente à exposição a campos magnéticos;

- Não existem dados experimentais ou teóricos sobre a densidade de corrente induzida nos tecidos e células que levam em consideração as variações locais das propriedades elétricas do meio (condutividade e permissividade);

- Os níveis de exposição de campos elétricos e outras características de campos magnéticos (harmônicos, transitórios, variações espaciais e temporais) têm recebido pouca atenção e não há muita informação disponível a respeito dos níveis de exposição a campos elétricos que não seja o valor médio quadrático da intensidade de campo; Não há evidências científicas convincentes de que a exposição a campos elétricos e magnéticos - $60 \mathrm{~Hz}$ - causa câncer em animais;

- Há evidências científicas de respostas comportamentais a campos elétricos e magnéticos (consideravelmente maiores do que aqueles encontrados em ambientes residenciais). Entretanto, efeitos neurocomportamentais adversos devido a campos extremamente elevados não foram comprovados. Evidências laboratoriais mostram que animais são capazes de detectar e responder a campos elétricos externos da ordem de $5 \mathrm{kV} / \mathrm{m}$. 
Diante dos estudos apresentados sobre os efeitos da exposição aos campos eletromagnéticos de uma LT, é necessário estabelecer os valores toleráveis a esses campos por órgãos regulamentadores. Os limites máximos de campos elétricos e magnéticos, gerados por linhas de transmissão de $60 \mathrm{~Hz}$, adotados pela Organização Mundial da Saúde (OMS) para a exposição ao público em geral, com base nas recomendações (ICNIRP, 1998), são de:

- Campo elétrico: $4,17 \mathrm{kV} / \mathrm{m}$;

- Densidade de fluxo magnético: $83,3 \mu \mathrm{T}$.

A ANEEL estipula que o valor máximo do campo elétrico, nos bordos da faixa, seja de $4,17 \mathrm{kV} / \mathrm{m}$ para o público em geral e $8,33 \mathrm{kV} / \mathrm{m}$ para o público ocupacional. A densidade de fluxo magnético limitado para o público em geral é de $200 \mu \mathrm{T}$ e $1000 \mu \mathrm{T}$ para o público ocupacional. (BRASIL, 2014).

\section{CONCLUSÕES}

Devido à notável relevância das LTs para o sistema de transmissão de energia elétrica, este trabalho apresenta os principais componentes e conceitos básicos existentes na operação de uma linha de transmissão em regime permanente. A avaliação dos níveis de campo elétrico e magnético é fundamental para garantir a segurança de pessoas e animais localizados na faixa de passagem destas linhas. Por isso, órgãos nacionais e internacionais estabelecem níveis máximos admissíveis para tais grandezas. Há um grande número de estudos e especulações sobre os efeitos da exposição de humanos aos campos eletromagnéticos; porém, nenhum deles apresenta resultados definitivos e concretos. Mas, mesmo assim, reforçam a relevância de estudos nessa área, buscando sempre determinar com eficácia os níveis de campos eletromagnéticos na faixa de servidão e corrigi-los, se for necessário. Para a determinação destes campos gerados por linhas de transmissão, sugerem-se estudos subsequentes de métodos analíticos para a elaboração de ferramentas que permitam o cálculo destes campos, para, assim, aplicá-los a planejamentos reais de sistemas de alta tensão. Estudos em otimização dos níveis eletromagnéticos, caso eles apresentem limites superiores propostos pelas normas regulamentadoras, também são de extrema valia. 


\section{ABSTRACT \\ CONCEPTUAL ANALYSIS OF TRANSMISSION LINES OPERATING IN STEADY SINUSOIDAL STATE}

This article presents the deportment of transmission lines operating in steady sinusoidal state and the effects caused by the electric (conductive and capacitive) and magnetic (inductive) couplings. The evaluation of the couplings is extremely important since they are directly associated with, for example, line losses, the safety and reliability of the interconnected electrical system and the safety of third parties when exposed to electromagnetic fields along the bonding range.

Keywords: Electromagnetic interference. Transmission lines. Electrical couplings. Magnetic couplings.

\section{REFERÊNCIAS}

BRASIL. Agência Nacional de Energia Elétrica. Resolução Normativa n. 616, de 1 de julho de 2014. Altera a Resolução Normativa n ${ }^{\circ} 398$, de 23 de março de 2010, que regulamenta a Lei $\mathrm{n}^{\circ} 11.934$, de 5 de maio de 2009 , no que se refere aos limites à exposição humana a campos elétricos e magnéticos originários de instalações de geração, transmissão e distribuição de energia elétrica, na frequência de 60 Hz. Brasília, 2014. Disponível em: <http://www2.aneel.gov.br/cedoc/ren2014616.pdf>. Acesso em: 01 set. 2015.

ASSOCIAÇÃO BRASILEIRA DE NORMAS TÉCNICAS. ABNT NBR 5422:1985: Projeto de linhas aéreas de transmissão de energia elétrica. Rio de Janeiro, 1985. 54 p.

COSTA, L. A. MONTEIRO, V. B. Minimização do campo magnético em linhas de transmissão, 2013. Disponível em: <http://www.cpdee.ufmg.br/ jramirez/disciplinas/ otimizacao/t3.pdf >. Acesso em: 12 set. 2015.

FUCHS, R. D. Transmissão de energia elétrica: linhas aéreas. Rio de Janeiro LTC, 1977. 588 p. v. 2.

GUEDES, T. S. Modelagem e medições de campos elétricos e magnéticos em linhas de transmissão. 2011. 109 f. Dissertação (Mestrado em Engenharia Elétrica) - Universidade Federal de Santa Catarina, Florianópolis, 2011. Disponível em: <http://repositorio.ufsc.br/ xmlui/handle/123456789/95171>. Acesso em: 12 set. 2015.

GUIMARÃES, G. E. Medições e cálculos de campos elétricos e magnéticos de uma linha de transmissão de 500 kV. 2005. 86 f. Dissertação (Mestrado em Engenharia Elétrica) Universidade Federal de Minas Gerais, Belo Horizonte, 2005. Disponível em: <http://www.ppgee.ufmg.br/defesas/456M.PDF>. Acesso em: 07 set. 2015. 
HAVAS, M. Biological effects of low frequency eletromagnetic fields. In: CLEMENTSCROOME, D. (Ed.). Electromagnetic environments and health in buildings. London: Taylor \& Francis, 2004. cap. 10.

HAYT, W. H. Eletromagnetismo. 3. ed. Rio de Janeiro: LTC, 1983. 254 p.

INTERNATIONAL COMMISSION ON NON-IONIZING RADIATION PROTECTION. (ICNIRP). Guidelines for limiting exposure to time-varying electric, magnetic, and electromagnetic fields (up to $300 \mathrm{GHz}$ ). Health Physics, v. 74, n. 4, p. 494-522, 1998. Disponível em: <http://www.icnirp.org/cms/upload/publications/ICNIRPemfgdl.pdf>. Acesso em: 12 set. 2015.

KUSTER, A. C. Avaliação de campos eletromagnéticos em regiões densamente povoadas. 2011. 96 f. Dissertação (Mestrado em Engenharia Elétrica) - Instituto de Tecnologia para o Desenvolvimento (LASTEC) Instituto de Engenharia do Paraná (IEP), Curitiba, 2011. Disponível em: <http://www.institutoslactec.org.br/menu-capacitacao/dissertacao-alvaroclaudino-kuster/>. Acesso em: 01 set. 2015.

LABEGALINI, P. R. et al. Projetos mecânicos das linhas aéreas de transmissão. 2. ed. São Paulo, Edgar Blucher, 1992. 528 p.

LIMA, G. F. Aula 05 - Apresentação da disciplina Instalações Elétricas de Alta Tensão Transmissão em Alta Tensão. Natal: Instituto Federal do Rio Grande do Norte, 2013.

MORENO, R. F. Campos eletromagnéticos e saúde humana: o fato e o mito. In: SEMINÁRIO NACIONAL DE PRODUÇÃO E TRANSMISSÃO DE ENERGIA ELÉTRICA, 18., 2005, Curitiba. Anais... Curitiba: SNPTEE, 2005.

MORENO, R. F. Possíveis efeitos sobre a saúde humana decorrentes da exposição a campos elétricos e magnéticos de baixa frequência. Revisão comentada da literatura. In: SEMINÁRIO NACIONAL DE PRODUÇÃO E TRANSMISSÃO DE ENERGIA ELÉTRICA, 16., 2001, Campinas. Anais... Campinas: SNPTEE, 2001.

OLIVEIRA, R. F. Linhas de transmissão. 2009. 48 f. Trabalho de Curso (Graduação em Engenharia Industrial Elétrica) - Universidade Federal do Paraná, Curitiba, 2009.

OPERADOR NACIONAL DO SISTEMA. Mapa do SIN. Disponível em: <http://www.ons.org.br/conheca_sistema/mapas_sin.aspx>. Acesso em: 12 out. 2015.

PEREIRA JUNIOR, C. A. Análise do transitório eletromagnético em linhas de transmissão. 2013. 78 f. Trabalho de Conclusão de Curso (Graduação em Engenharia Elétrica) - Instituto Federal de Goiás, Campus Jataí, Jataí, 2013. Disponível em: <http://ifgjatai.webcindario.com/CarlosAPJ.pdf>. Acesso em: 01 set. 2015.

PERRO, B. S. Estudo dos campos eletromagnéticos em linhas de transmissão a frequência industrial. 2007. 63 f. Trabalho de Conclusão de Curso (Graduação em Engenharia Elétrica) - Escola Politécnica, Universidade Federal do Rio de Janeiro, Rio de Janeiro, 2007. Disponível em: <http://monografias.poli.ufrj.br/monografias/ monopoli10001334.pdf>. Acesso em: 01 set. 2015. 
POLK, C. Biological effects of low-level low-frequency electric and magnetic fields. IEEE Transactions on Education, v. 34, n. 3, p. 243-249, ago. 1991.

RAMÍREZ, J. A. Campos eletromagnéticos devido a sistemas de energia elétrica. In: WORKSHOP NACIONAL: EFEITOS BIOLÓGICOS DEVIDOS À AÇÃO DE CAMPOS ELETROMAGNÉTICOS, São Paulo. Anais... São Paulo: USP, 2001. 24 p. Disponível em: <http://www.cpdee.ufmg.br/ jramirez/publicacoes/acn2001.pdf>. Acesso em: 01 set. 2015.

SADIKU, M. N. O. Elementos do Eletromagnetismo. Porto Alegre: Bookman, 2004.

SANTOS, M. G. Acoplamento elétrico entre linhas de transmissão operando em regime permanente e dutos metálicos aéreos. 2011. 129 f. Dissertação (Mestrado em Engenharia Elétrica) - Universidade Federal de São João Del-Rei, São João Del-Rei, 2011. Disponível em: <http://www.ppgel.ufsj.edu.br/portal/images/stories/Dissertacoes/

DISSERTACAOVERSAOFINAL.pdf>. Acesso em: 01 set. 2015.

WERTHEIMER, N.; LEEPER, E. Electrical wiring configurations and childhood cancer. Am. J. Epidemiol, v. 109, n. 3, p. 273-84, 1979.

Recebido em: 31/08/2016

Aprovado em: 18/10/2016

Publicado em: 02/12/2016 